\title{
(6) OPEN ACCESS \\ Reconsidering the back door approach by targeting the coronary sinus in ischaemic heart disease
}

\author{
Giovanni Luigi De Maria, ${ }^{1,2}$ George Kassimis, ${ }^{3}$ Tushar Raina, ${ }^{3}$ Adrian P Banning ${ }^{1}$
}

${ }^{1}$ Department of Cardiology, John Radcliffe Hospital, Oxford University Hospitals NHS Foundation Trust, Oxford, UK ${ }^{2}$ Department of Cardiovascular Medicine, Catholic University of the Sacred Heart, Policlinico

A. Gemelli, Rome, Italy ${ }^{3}$ Department of Cardiology, Cheltenham General Hospital, Gloucestershire Hospitals NHS Foundation Trust, Cheltenham, UK

\section{Correspondence to} Professor Adrian P Banning, Consultant Interventional Cardiologist, Department of Cardiology, John Radcliffe Hospital, Oxford University Hospitals NHS Foundation Trust, Headley Way, Oxford OX3 9DU, UK:

Adrian.Banning@ouh.nhs.uk

Received 14 March 2016 Revised 21 March 2016 Accepted 9 April 2016 Published Online First 10 June 2016
CrossMark

\author{
To cite: De Maria GL, \\ Kassimis G, Raina T, et al. \\ Heart 2016;102:
}

1263-1269.

\section{ABSTRACT}

Coronary sinus interventions (CSI) are a class of invasive techniques (surgical and percutaneous) originally proposed in the first half of the 20th century, aiming to treat ischaemic heart disease by acting on the venous coronary system. Three main classes of CSI have been proposed and tested: (1) retroperfusion technique, (2) retroinfusion technique and (3) coronary sinus occlusion techniques. They all share the principle that a controlled increased pressure within the coronary sinus may promote a retrograde perfusion of the ischaemic myocardium with consequent cardioprotection. Development of arterial treatments including coronary aortic bypass grafting and then percutaneous coronary intervention deflected interest from interventions on the coronary venous system. However, CSI may still have a possible niche role today in specific and selected clinical contexts in which existing therapies are insufficient. In this review paper, we aim to revise the rationale for $\mathrm{CSI}$, describing the details and the evidence collected so far about these techniques and to provide insights about the main clinical scenarios in which these strategies may find a contemporary application in combination or as an alternative to existing approaches.

\section{INTRODUCTION}

Prompt coronary revascularisation by cardiac surgery or percutaneous coronary intervention (PCI) has dramatically contributed to a reduction in short-term mortality and improved quality of life for many patients presenting with ischaemic heart disease (IHD). However, this improved efficacy of the initial treatment for patients with IHD in combination with a longer expectancy of life is evolving a new demography of patients-who are older and with more comorbidities including heart failure and often with a history of previous coronary interventions (both PCI and coronary artery bypass grafting $(\mathrm{CABG}))$. These patients are likely to require new treatment approaches and novel therapeutic strategies.

Over the last 60 years, the therapeutic approach to IHD has been dominated by the assumption that optimal myocardial perfusion can be restored by improving antegrade blood flow down the coronary artery system. However, at least two decades before the first CABG operation in 1964 and three decades before the first angioplasty in 1979 a totally different approach was proposed to provide blood to the ischaemic myocardium by altering the venous return of the heart. This concept is the underlying principle behind 'coronary sinus interventions' (CSI) aiming to protect the ischaemic myocardium by acting on the so-called 'back door to the heart'. ${ }^{1}$
In this review paper, we aim to analyse the rationale for CSI, describing the details and the evidence available for the main CSI techniques, providing insights about the main clinical scenarios in which these new approaches may find a contemporary application in combination or as an alternative to conventional strategies.

\section{CSI: the rationale}

Three main categories of CSI have been described and adopted over the last century: retroperfusion, retroinfusion and coronary sinus occlusion ${ }^{2}$ (figure 1 ). Retroperfusion technique aimed to provide arterialised blood to the ischaemic myocardium via the coronary vein system. ${ }^{2}$ Retroinfusion technique aimed to deliver drugs to the myocardium essentially for cardioprotection, by a retrograde approach involving the access to the coronary sinus. ${ }^{2}$ Coronary sinus occlusion techniques aimed to support myocardium perfusion without active infusion of arterialised blood or drugs, but essentially slowing down the outflow of arterialised blood from the heart, obeying the principle 'if you cannot increase coronary input, let's decrease its output'. ${ }^{2}$ Coronary sinus occlusion techniques can be categorised as balloon-based or 'temporary coronary sinus occlusion techniques' and non-balloon-based or 'permanent coronary sinus occlusion techniques'.

The concept of using the vein system as a way to guarantee myocardial perfusion in the presence of coronary artery disease is attractive for three principal reasons: (1) the coronary vein system has a very high volume accounting for $2 / 3$ of the whole intracoronary blood volume, with a venous microcirculatory surface area six times larger than the surface area of the arterial capillary bed; (2) the coexistence of three interconnected venous systems (the coronary sinus, the anterior cardiac veins and the Thebesian system) with an extremely dense meshwork; (3) the coronary vein system appears to be resistant to atherosclerosis even when there is diffuse coronary arterial disease. $^{2}$

Interestingly, if the heart had a single rather than an interdigitated venous system (as present in most other organs and tissues), an increase in pressure in the coronary sinus would lead to blood stagnation and intramyocardial haemorrhage. ${ }^{3}$ In man, a controlled increase of pressure in the coronary sinus is associated with increased collateral flow going to the ischaemic territory. ${ }^{4}$ This occurs as a consequence of a shift of oxygenated blood from the remote myocardium towards the ischaemic territory (figure 2) and opening of arterovenous anastomosis and intravenous interconnections. These connexions are between the coronary sinus, the 


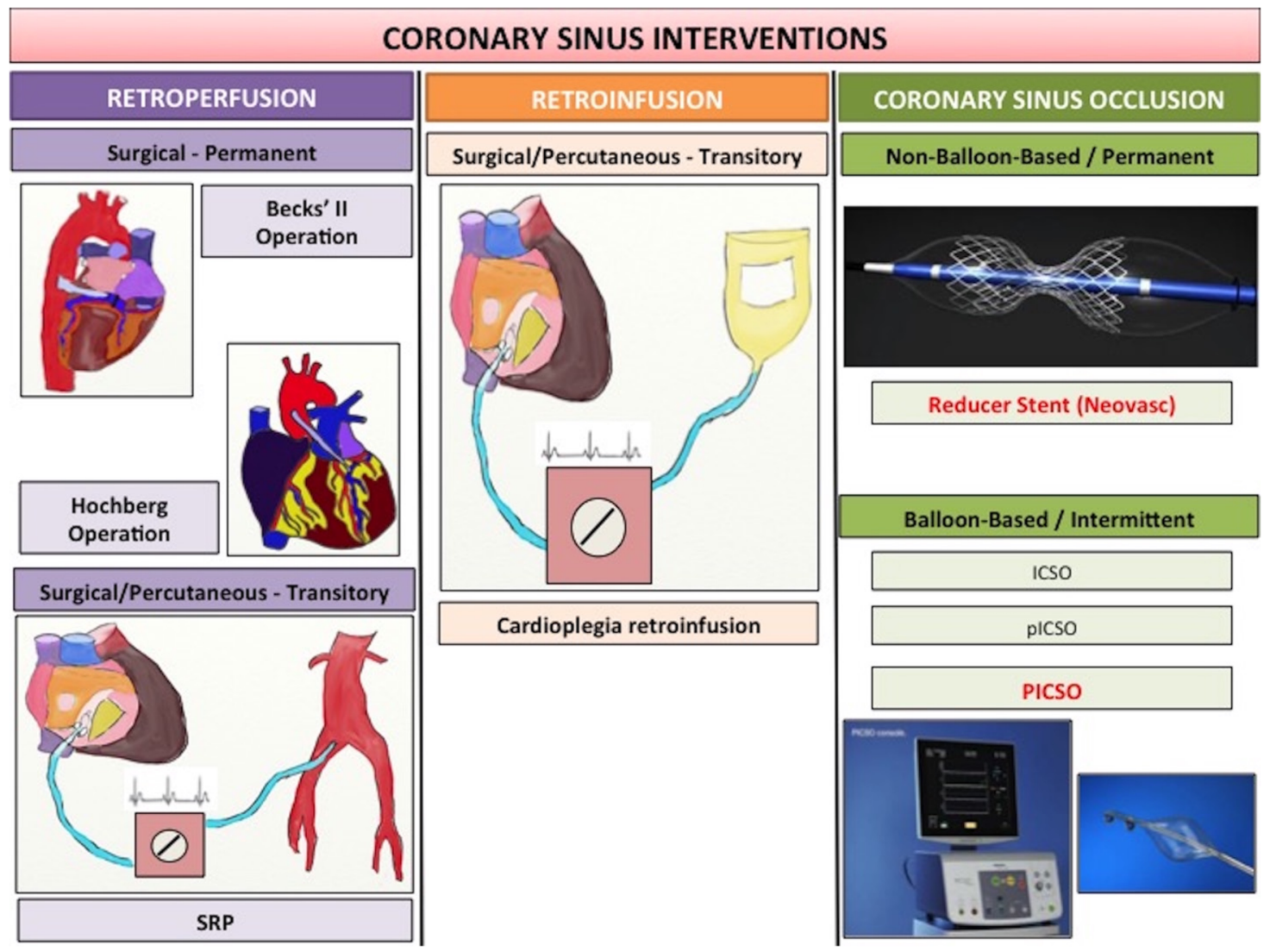

Figure 1 Classification of coronary sinus interventions. ICSO, intermittent coronary sinus occlusion; pICSO, pressure-observed coronary sinus occlusion; PICSO, pressure-controlled coronary sinus occlusion; SRP, synchronised retroperfusion.

Thebesian system and the venous plexus. ${ }^{3}$ Notably, the location of the venous plexus and the Thebesian system, mainly at the endocardial level, ${ }^{5}$ is particularly responsible for a redistribution of the pressure perfusion gradient from the endocardium to the epicardium reversing the ischaemic wavefront phenomenon ${ }^{6}$ (figure 2).

Importantly, the efficacy of any CSI is related to the ability to produce a controlled increase in pressure within the coronary
Figure 2 Mechanism of action of coronary sinus interventions. Increase in collateral flow towards the ischaemic area from the remote myocardium through activation of artero-artero (*)and veno-venous ( $¥)$ connexions as a consequence of raised pressure within the coronary sinus (A and B) Blood flow directions are highlighted by the arrows in the panels. Increase in coronary sinus pressure produces also a redistribution of flow favouring the subendocardium layer with increased endocardium/ epicardium flow ratio, through connexions between the coronary sinus system and the Thebesius venous system and venous plexus highly represented within the endocardium (C and D).

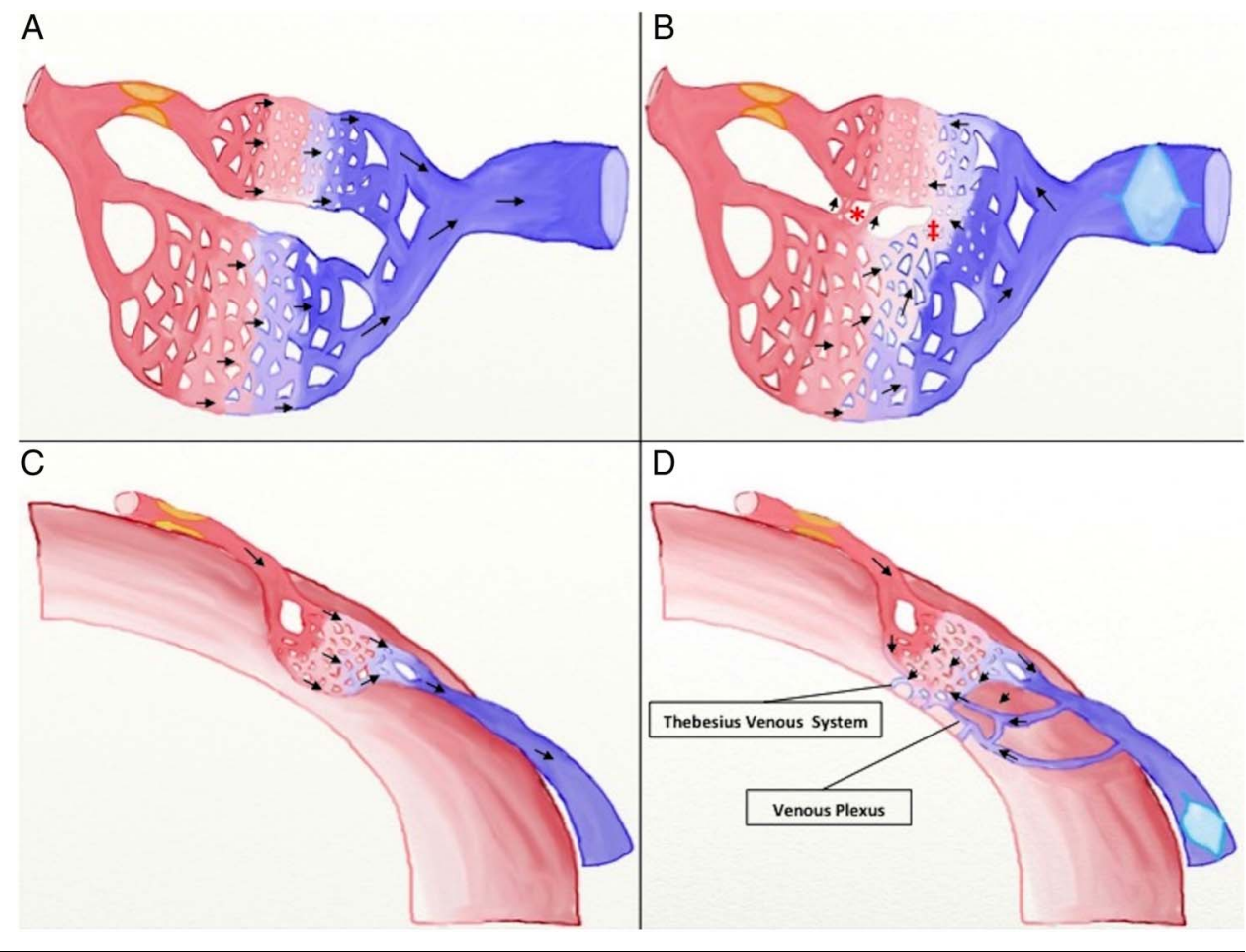


sinus to preserve coronary artery inflow and the physiologic systolic cardiac venous drainage. ${ }^{2}$

\section{TECHNIQUES FOR CSI Retroperfusion}

The idea of perfusing the ischaemic heart backwards from the coronary sinus was proposed for the very first time at the end of the XIX century by Pratt in a feline model and then confirmed by Claude Beck and collaborators in 1949 with the first attempt of coronary venous bypass grafting. ${ }^{7}$ The Beck II intervention consisted of a vascular graft between the descending aorta and the coronary sinus followed few weeks after an operative reduction in diameter of the ostium of the coronary sinus.

The immediate results associated with this approach were initially encouraging with an immediate $6 \%$ postoperative mortality in a first series of 347 patients and with a reduced occurrence of ventricular fibrillation in patients treated. ${ }^{8}$

However, an unexplained 25\% long-term mortality was observed with the technique. ${ }^{9}$ In retrospect it is clear that permanent obstruction to the coronary sinus used in the Beck II intervention resulted in detrimental considerable and permanent increase in intraluminal pressure with constant obstacle to systolic outflow with consequent development of intramyocardial oedema and haemorrhage. ${ }^{10}$

A second attempt of surgical retroperfusion was the selective arterialisation of a coronary vein proposed by Hochberg et al. ${ }^{11}$ The intervention consisted in placing a venous graft between the aorta and a coronary vein which was ligated distally. This Beck II intervention resulted in a constant and significant increase in coronary vein pressure with consequent impairment of coronary artery inflow. Consequently, ventricular function was compromised and clinical outcomes were disappointing.

A true step forward in retroperfusion technique occurred in the 1970s after the pioneering studies by Meebaum, Markov and Wiener who described the concept of diastole synchronised retroperfusion (SRP). ${ }^{2}$ The retroperfusion system consisted of an electronic pumping console connected to an $8 \mathrm{~F}$ catheter with multiple side holes placed in the femoral artery and an $8.5 \mathrm{~F}$ catheter with an inflatable balloon at $1 \mathrm{~cm}$ from its tip placed in the coronary sinus. During diastole, the balloon was inflated and arterial blood, aspirated via the arterial catheter, was actively infused into the coronary sinus. During systole, the infusion stopped and the balloon was deflated to allow coronary venous drainage.

Animal models have confirmed the efficacy of SRP in improving myocardial metabolism, left ventricular function with a concomitant reduction of the ischaemic zone and infarct size. ${ }^{12} 13$ These initial encouraging results with SRP were replicated in man and a potential role in supporting elective high-risk angioplasty to the left anterior descending artery was suggested in the prestent era. ${ }^{14}$ However, these retroperfusion approaches failed to enter clinical practice because of their technical complexity and the requirement for cumbersome devices with sophisticated pump systems to strictly control retrograde flow.

\section{Retroinfusion}

Retroinfusion approaches are overall similar to retroperfusion techniques, differing in the fact that retroinfusion techniques aim to deliver drugs and not arterialised blood to the heart. For this reason, its main application has occurred in the cardiac surgery field to deliver cardioplegia during cardiac interventions.

Because of more homogenous distribution of the cardioplegia, improved myocardial protection has been proposed for a retrograde delivery of cardioplegia compared with the anterograde approach. $^{2}$ Lillehei et al ${ }^{15}$ highlighted a further advantage of retroinfusion in surgery for aortic regurgitation or in interventions requiring the opening of the aortic root, since retroinfusion still allowed a complete and homogenous delivery of cardioplegia without putting coronary arteries ostia at risk.

Limitations of retroinfusion include limited protection for the right ventricle, whose venous drainage is not entirely dependent on the coronary sinus venous system, ${ }^{2}$ and a delayed onset of cardiac arrest compared with the conventional anterograde approach. ${ }^{2}$ For this reason, a combined anterograde and retrograde delivery of cardioplegia by retroinfusion has been suggested.

\section{Coronary sinus occlusion techniques}

Balloon-based/intermittent coronary sinus occlusion techniques

The principle that an intermittent and pressure-controlled increase in coronary sinus pressure is pivotal for the success of a CSI is fully embodied by the techniques of intermittent coronary sinus occlusion (ICSO).

Because of the large capacity volume of the cardiac venous system, it is unlikely that a single diastole-lasting balloon occlusion can produce a consistent increase in coronary sinus pressure. The first example of ICSO was proposed in 1977 consisting of a balloon-induced coronary sinus occlusion at a predefined rate of 60 balloon inflation/deflations $/ \mathrm{min}^{2}{ }^{2}$ The limitation of this first attempt was the duration of the inflation/deflation cycle, which was arbitrary-defined without any guarantee that either balloon deflation occurred within systole allowing drainage of the venous system or that optimal coronary sinus pressure was achieved.

The natural evolution to this approach was the technique proposed by Mohl et $a l^{16}$ and essentially it consisted in regulating the span of the balloon inflation/deflation cycle according to ECG and coronary sinus pressure. This technique was designated as pressure-observed intermittent coronary sinus occlusion (pICSO) and then upgraded into pressure-controlled intermittent coronary sinus occlusion (PICSO). pICSO and PICSO share the same basic principle of pressure-controlled coronary sinus occlusion, with the only difference that coronary sinus pressure and balloon activation is completely automated in PICSO while manually controlled by the operator in pICSO.

In both pICSO and PICSO treatments, a balloon-tipped catheter equipped with a sensor for coronary sinus pressure monitoring is placed at the ostium of the coronary sinus. During balloon inflation, a pulsatile and progressive increase in coronary sinus pressure is observed at each cardiac cycle until a pressure plateau is achieved. At this stage, the balloon is deflated (manually in pICSO, automatically in PICSO) allowing systolic venous drainage.

The achievement of a plateau of coronary sinus pressure can be interpreted as a sign of a completed redistribution of blood flow within the coronary venous system, favouring the increased collateral flow towards the ischaemic territory and the desirable redistribution of endocardium/epicardium blood flow ratio. ${ }^{17}$

Compared with all the other CSI, the potential myocardial salvage associated with ICSO techniques and PICSO, in particular, may be related to two further mechanisms of action on top of the well-described redistribution of blood flow towards the ischaemic area and the endocardium.

The first of these two mechanisms is represented by the so-called ability of PICSO to wash out fluid (reducing oedema) and noxious soluble factors from the area at risk $^{17}$ (figure 3 ). After the results of studies on coronary sinus blood density during ICSO treatment, Mohl et $a l^{18}$ suggested indeed that the rapid reduction of coronary sinus pressure after the prolonged 
Figure 3 Peculiar mechanisms of action of intermittent coronary sinus occlusion strategies and especially of pressure-controlled coronary sinus occlusion (PICSO). Depression within the coronary sinus following balloon deflation produces a washout of interstitial fluids (oedema) and removal of toxic metabolites released by ischaemic myocardium (A and $B$ ). According to the 'embryonic recall' theory, pulsatile increased pressure within the coronary sinus leads to increased shear stress acting on the endothelium of venous vasculature, with modulation of genomic transcription and upregulation of cardioprotective, pro-agiogenetic and regenerative pathways (C). $\mathrm{HO}-1$, haeme-oxygenase 1; VEGF-1, vascular endothelial growth factor-1.
A
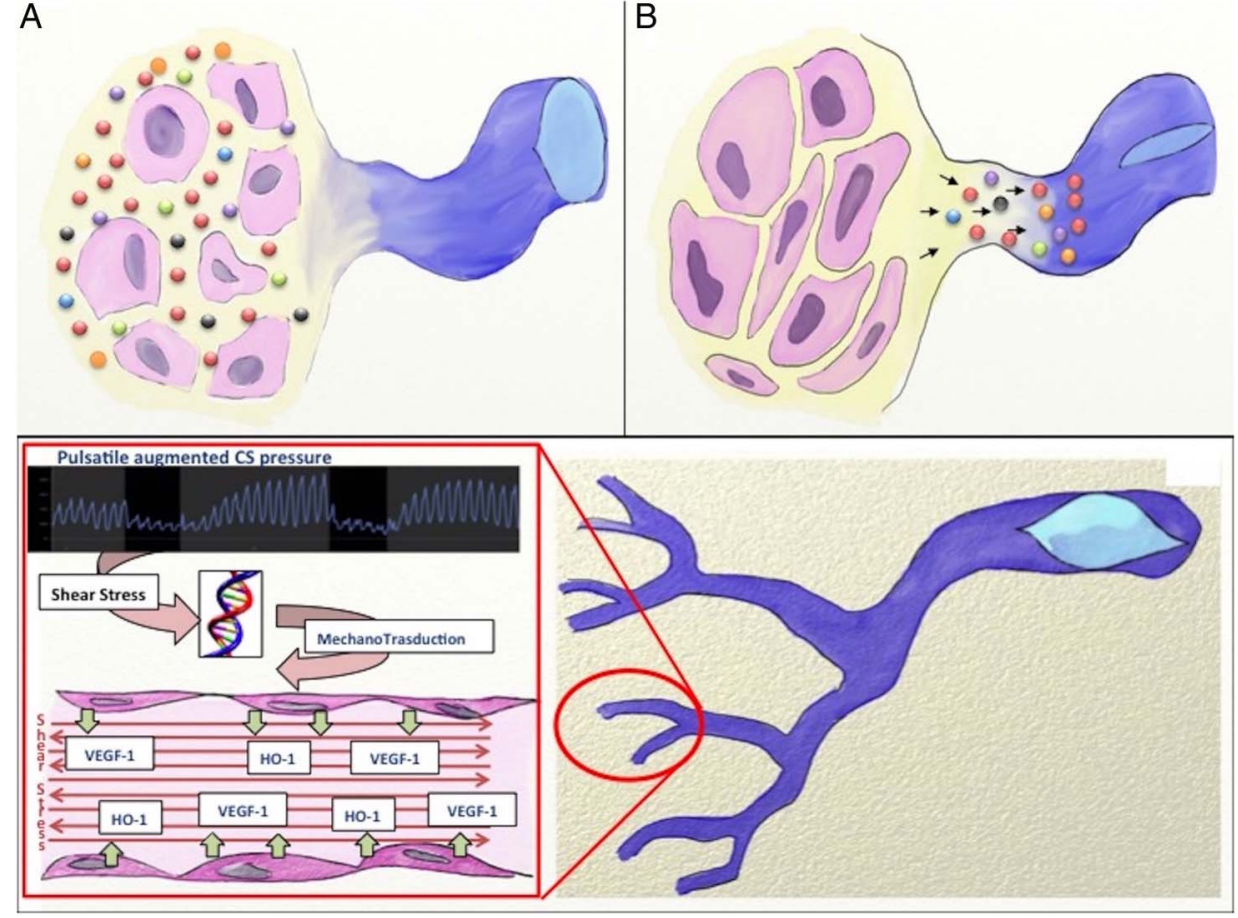

phase of plateau may induce a sort of aspirating effect on fluids and toxic metabolites accumulated in the ischaemic segment. In this way, PICSO would not just allow, but would improve the systolic cardiac venous drainage.
A further potential mechanism to explain the benefit of ICSO techniques and PICSO in particular has been highlighted by Weigel et $a l,{ }^{19}$ who described a significantly increased upregulation of haeme-oxygenase-1 and vascular endothelial growth
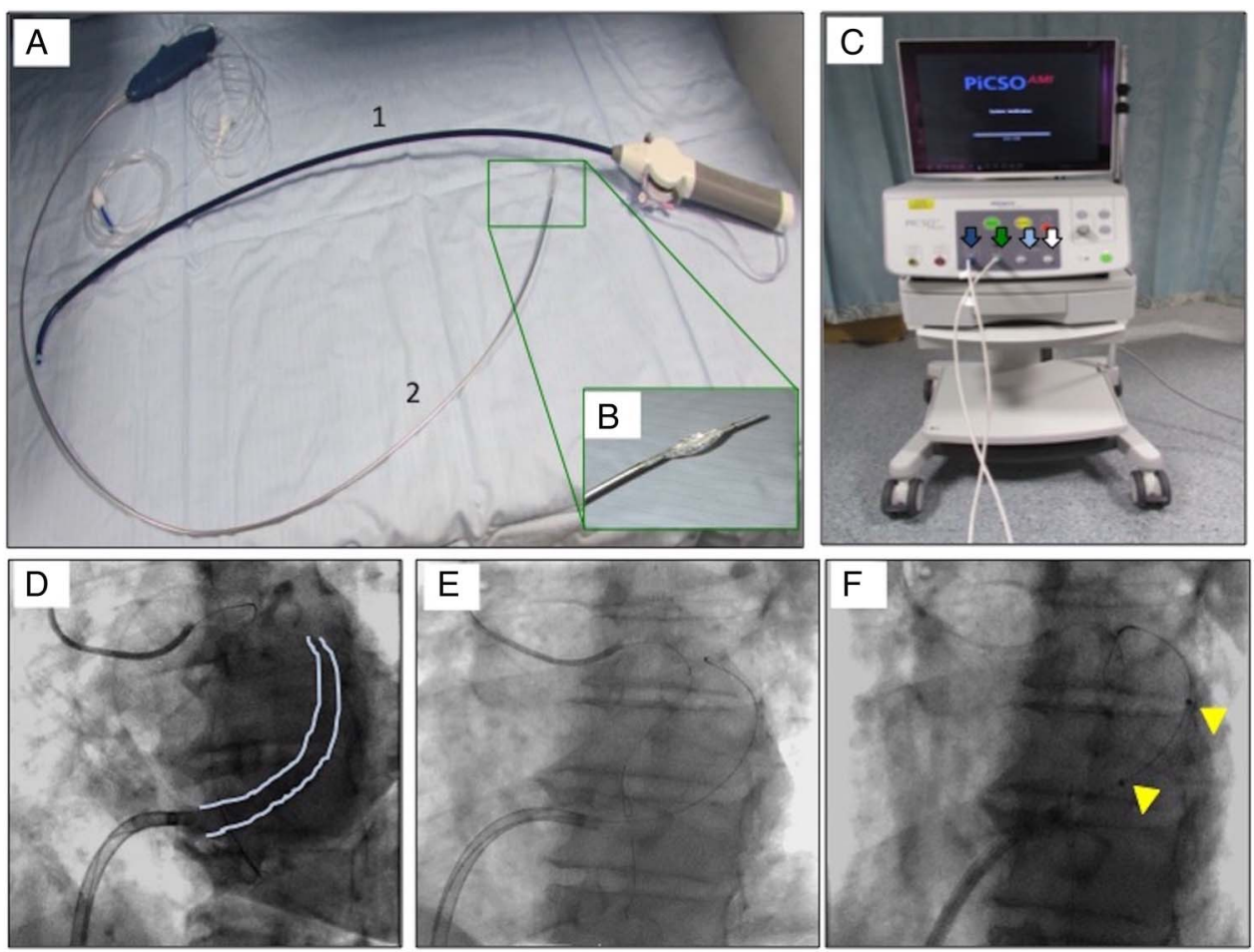

Figure 4 Components of the pressure-controlled coronary sinus occlusion (PICSO) system and procedural steps. (A) Steerable venous guidesheath (external lumen 10F) for coronary sinus cannulation (1) and double lumen PICSO balloon catheter (2) with details of balloon profile (B). (C) PICSO consoles with highlighted connexions for coronary sinus pressure reading (blue arrow), ECG monitoring (green arrow) and helium inlet and outlet for PICSO balloon inflation and deflation (blue and white arrows). Coronary sinus is cannulated after 'roadmapping' its position during the venous phase of a coronary angiogram in left anterior oblique (LAO) view (coronary sinus profile highlighted by blue lines) (D). After wiring with $0.032^{\prime \prime}$ hydrophilic wire (E), PICSO balloon is advanced in the so-called 'silent region' of the coronary sinus (F, with yellow arrowheads highlighting the edges of the deflated balloon) to achieve occlusion of all main coronary sinus side branches during balloon inflation. 


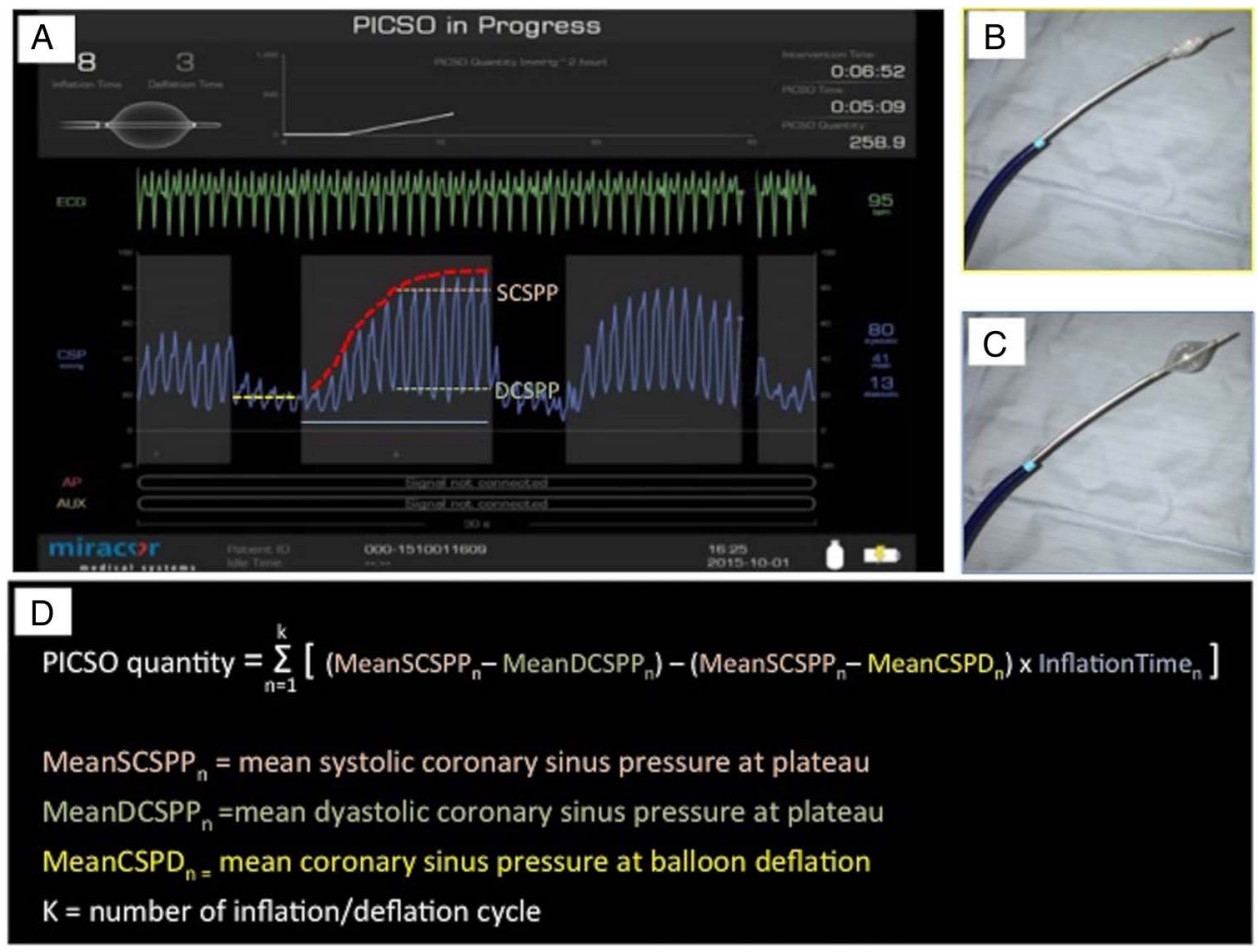

Figure 5 Coronary sinus pressure traces during pressure-controlled coronary sinus occlusion (PICSO) (A). Highlights the coronary sinus pressure trend during PICSO treatment with the corresponding balloon profiles during inflation and deflation (B and C). To note the achievement of a plateau (dotted red line) of coronary sinus pressure during balloon inflation. (D) Reports the formula for PICSO quantity calculation proposed by Miracor Medical Systems.

factor 1 gene transcription in the infarcted region of PICSO-treated animals. This observation, further corroborated by reports describing the effect of mechanical shear stress over gene regulation in endothelial cells, ${ }^{20}$ has raised the possibility that PICSO may promote gene reprogramming and favour myocardium self-protection and regeneration ${ }^{21}$ (figure 3 ).

The preliminary results suggesting cardioprotection effect of ICSO techniques (ICSO, pICSO and PICSO) with a reproducible reduction in infarct size ${ }^{22}$ observed in animal models have been subsequently confirmed in small clinical studies, in which PICSO was tested in patients with ST-elevation myocardial infarction (STEMI) in both thrombolysis and primary PCI eras. ${ }^{23} 24$

The Prepare RAMSES study has recently tested PICSO in patients with anterior STEMI. ${ }^{25}$ The study was too small in size and with a lower than expected rate of PICSO treatment delivered, to draw any definite conclusions. However, it showed a significant higher shrinkage of the infarct size at 4 months follow-up in the treated group compared with controls. More importantly, the study showed a correlation between the degree of PICSO therapy delivered and infarct size reduction. ${ }^{25}$ The results of the study have triggered the introduction of a newer and more stable balloon catheter and the development by Miracor Medical Systems of an automated algorithm for a constant monitoring of PICSO quantity delivered to the patient (figures 4 and 5).

\section{Non-balloon-based/permanent coronary sinus occlusion} techniques

The attention to this type of techniques has been triggered by the publication of the coronary sinus reducer for treatment of refractory angina (COSIRA) trial. ${ }^{26}$ The study randomised 104 patients with refractory angina and unsuitable for revascularisation to implantation of an hourglass-shaped stainless steel balloon-expandable stent in the coronary sinus (Reducer, Neovasc, Vancouver, Canada) versus sham controls. The Reducer stent is designed to produce a controlled narrowing of the coronary sinus, and its nominal diameter ranges from 7 to $13 \mathrm{~mm}$ at the edges and is $3 \mathrm{~mm}$ at its mid-portion.

The COSIRA study extended the preliminary results of previous feasibility-safety studies, ${ }^{27}$ showing an improvement of two Canadian Cardiovascular Society Angina classes in 35\% of patients in the Reducer group versus the $15 \%$ of patients in the control group ( $\mathrm{p}$ : 0.02), with a significant improvement in quality of life assessed by the Seattle Angina Questionnaire. ${ }^{26}$

Longer term outcome is awaited especially as the Reducer stent might produce a permanent change in the anatomy of the coronary vein system, potentially exposing to the risk of an uncontrolled rise in pressure within the coronary sinus, which has been identified as the common denominator underlying the initial failure of the surgical permanent retroperfusion techniques (Beck II and Hochberg interventions).

\section{CLINICAL SETTING FOR POTENTIAL SUCCESSFUL APPLICATIONS OF CSI}

Because of their simplicity, among the CSI techniques, it is more likely that coronary sinus occlusion techniques, namely the Reducer stent and PICSO, may find a future role and currently at least three scenarios could be considered for future applications of CSI: refractory angina, STEMI and PCI in complex coronary anatomy/high-risk patients.

\section{Refractory angina}

The results of the COSIRA study have stoked enthusiasm into the application of CSI in the treatment of patients with refractory angina who are not candidates for revascularisation. ${ }^{26}$ If the results of the study are corroborated by future data, confirming 
Method\#1 (preferable approach in LAO $30^{\circ}$ )
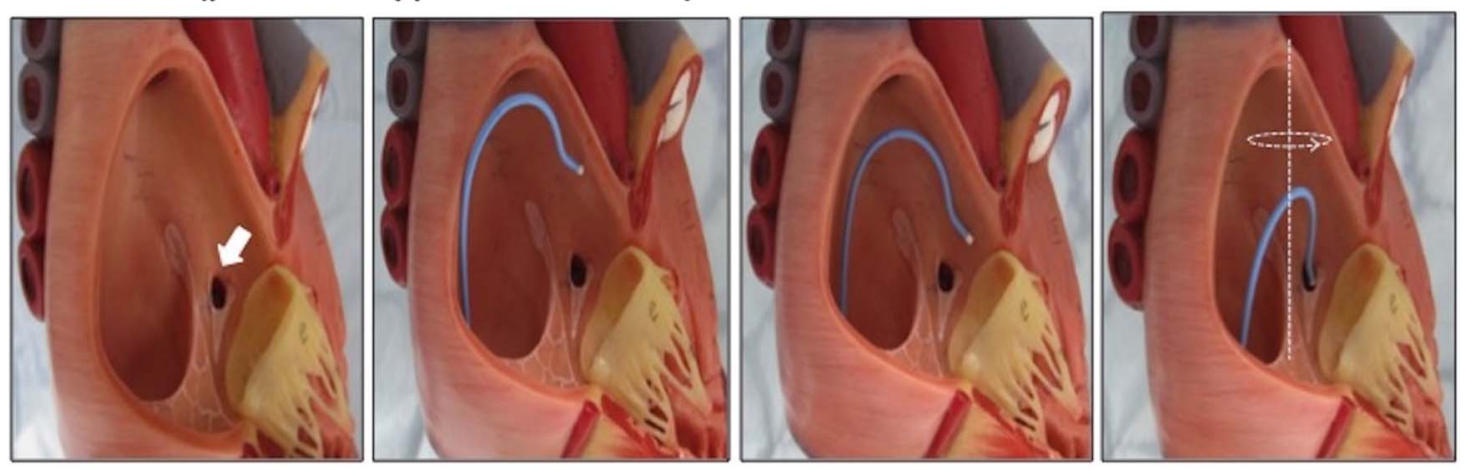

\section{Method\#2 (preferable approach in RAO $30^{\circ}$ )}
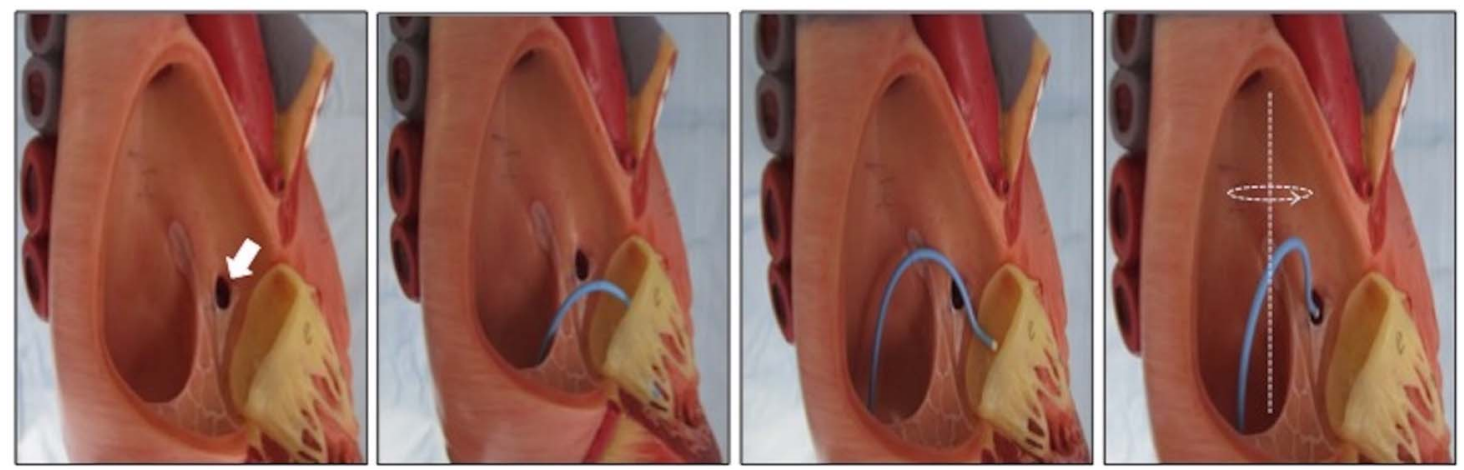

Figure 6 Two main techniques for coronary sinus cannulation. Method \#1 is typically performed in LAO $30^{\circ}$ view. The catheter for coronary sinus cannulation (eg, an Amplatz left 1) is pulled down from the roof of the right atrium until the tricuspid plane following the profile of the interatrial septum and performing a counterclockwise rotation posteriorly to engage the ostium of the coronary sinus (white arrow). Method \#2 is typically performed in right anterior oblique (RAO) $30^{\circ}$ view. The tricuspid valve is first crossed and then the catheter for coronary sinus cannulation is gently pulled back performing a counterclockwise rotation posteriorly to engage the ostium of the coronary sinus (white arrow).

safety and efficacy, then the Reducer stent could represent a new weapon in the armamentarium of interventional cardiologists.

The chronic nature of refractory angina condition combined with the actual intermittent mode of action provided by PICSO makes it a less attractive option in this category of patients.

\section{ST-elevation myocardial infarction}

Though primary PCI has dramatically reduced the in-hospital and long-term mortality associated with this condition, it does remain a variable proportion of patients in whom restoration of epicardial coronary artery patency does not translate into effective myocardial reperfusion. This condition is well known as no reflow and is mainly related to a profound impairment of coronary microvasculature secondary to ischaemia/reperfusion injury and distal embolisation. ${ }^{28}$ The advocated mechanisms of action of PICSO make it a very attractive device for application in limiting and eventually minimising the impact of no reflow. The results of the Prepare RAMSES study are encouraging but more evidence is required. ${ }^{25}$ Ongoing clinical trials using PICSO are exploring the potentialities of this device in this specific setting.

\section{$\mathrm{PCl}$ in complex anatomy/high-risk patients}

Supporting the systemic and the coronary circulation and reducing the left ventricle workload are the main goals of all percutaneous circulatory assist devices as intraortic balloon pump (IABP), Impella, Tandem Heart and extracorporeal membrane oxygenation (ECMO).

Interestingly in animal models, actual coronary flow provided by mechanical support is nearly comparable to the baseline coronary flow in the absence of stenosis, with some reports showing no increase in coronary blood flow at all beyond the stenosis. $^{29}$ This may allow consideration of CSI, and PICSO mainly, in implementing myocardial support by further reducing ischaemic burden in PCI on high-risk patients/complex coronary anatomy. Additionally, because of its transvenous insertion, PICSO application would limit the risk of bleeding and access site-related complications.

PICSO cannot support the circulation in the management of patients in cardiogenic or precardiogenic shock, but a combined approach with devices like IABP or Impella might produce more effective cardiac recovery in high-risk patients. ${ }^{30}$ PICSO might find an even easier application in patients on ECMO, allowing a certain degree of cardioprotection otherwise not provided by ECMO which is known to pay the outstanding systemic haemodynamic support with an increased left ventricular afterload, higher wall stress and greater myocardial oxygen demand. ${ }^{29}$

Beside an intraprocedural application, it is also plausible that pretreating patients with PICSO before PCI might improve their tolerance to transient PCI-induced ischaemia potentially preventing the need for upfront application of more complex circulatory support devices.

\section{FUTURE PERSPECTIVES}

After a first tide of enthusiasm and arguably nearly three decades of neglect, CSI might yet be revived. Results from the ongoing clinical trials will clarify if these techniques may find an established role in the management of IHD. If efficacy is confirmed, coronary sinus cannulation will be a required skill in the 
portfolio of interventional cardiologists (figure 6). However, in the current era, the real challenge will be to balance the potential benefit deriving from these techniques with their cost in terms of both money and time, putting naturally patient's safety and interest first.

Contributors All authors have provided substantial contributions to (1) the conception and design of the work, or the acquisition; (2) drafting the work or revising it critically for important intellectual content; (3) have given final approval of the version published; (4) and agree to be accountable for all aspects of the work in ensuring that questions related to the accuracy or integrity of any part of the work are appropriately investigated and resolved.

Competing interests APB is chief investigator of the investigators-initiated OxAMI PICSO Study (15/SC0167), supported NIHR-BRC Oxford and Miracor Medical Systems. GLDM is co-investigator of the OxAMI-PICSO study.

Patient consent Obtained.

Provenance and peer review Not commissioned; externally peer reviewed.

Open Access This is an Open Access article distributed in accordance with the Creative Commons Attribution Non Commercial (CC BY-NC 4.0) license, which permits others to distribute, remix, adapt, build upon this work non-commercially, and license their derivative works on different terms, provided the original work is properly cited and the use is non-commercial. See: http://creativecommons.org/ licenses/by-nc/4.0/

\section{REFERENCES}

1 Mohl W, Milasinovic D, Maurer G. Transcoronary sinus catheter interventions: back in the repertoire? Eurolntervention 2015;11:19, 21, 23.

2 Mohl W. Coronary sinus interventions: from concept to clinics. J Card Surg 1987;2:467-93.

3 Kassab GS, Navia JA, March K, et al. Coronary venous retroperfusion: an old concept, a new approach. J Appl Physiol 2008;104:1266-72.

4 Sato M, Saito T, Mitsugi M, et al. Effects of cardiac contraction and coronary sinus pressure elevation on collateral circulation. Am J Physiol 1996;271:H1433-40.

5 Tsang JC, Chiu RC. The phantom of "myocardial sinusoids": a historical reappraisal. Ann Thorac Surg 1995;60:1831-5.

6 Ido A, Hasebe N, Matsuhashi $\mathrm{H}$, et al. Coronary sinus occlusion enhances coronary collateral flow and reduces subendocardial ischemia. Am J Physiol Heart Circ Physiol 2001;280:H1361-7.

7 Becks CS, Stanton E, Batiuchok W, et al. Revascularization of the heart by graft of systemic artery into coronary sinus. J Am Med Assoc 1948;137:436-42.

8 Eckestein RW, Hornberger JC, Sano T. Acute effects of elevation of coronary sinus pressure. Circulation 1953;7:422-36.

9 Jones DS. The puzzle of positive results-myocardial revascularization. N Engl J Med 2015:372:501-3.

10 Hahn RS, Kim M. Revascularization of the heart; histologic changes after arterialization of the coronary sinus. Circulation 1952:5:810-15.

11 Hochberg MS, Roberts WC, Morrow AG, et al. Selective arterialization of the coronary venous system. Encouraging long-term flow evaluation utilizing radioactive microspheres. J Thorac Cardiovasc Surg 1979;77:1-12.
12 Meerbaum S, Lang TW, Osher JV, et al. Diastolic retroperfusion of acutely ischemic myocardium. Am J Cardiol 1976;37:588-98.

13 O'Byrne GT, Nienaber CA, Miyazaki A, et al. Positron emission tomography demonstrates that coronary sinus retroperfusion can restore regional myocardial perfusion and preserve metabolism. J Am Coll Cardiol 1991;18: 257-70.

14 Incorvati RL, Tauberg SG, Pecora MJ, et al. Clinical applications of coronary sinus retroperfusion during high risk percutaneous transluminal coronary angioplasty. J Am Coll Cardiol 1993;22:127-34.

15 Lillehei CW, Dewall RA, Gott VL, et al. The direct vision correction of calcific aortic stenosis by means of a pump-oxygenator and retrograde coronary sinus perfusion. Dis Chest 1956:30:123-32.

16 Mohl W. The development and rationale of pressure-controlled intermittent coronary sinus occlusion-a new approach to protect ischemic myocardium. Wien Klin Wochenschr 1984;96:20-5.

17 Mohl W, Gangl C, Jusić A, et al. PICSO: from myocardial salvage to tissue regeneration. Cardiovasc Revasc Med 2015;16:36-46.

18 Mohl W, Punzengruber C, Moser M, et al. Effects of pressure-controlled intermittent coronary sinus occlusion on regional ischemic myocardial function. J Am Coll Cardiol 1985;5:939-47.

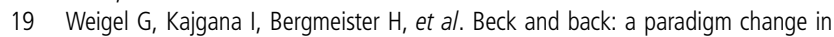
coronary sinus interventions-pulsatile stretch on intact coronary venous endothelium. J Thorac Cardiovasc Surg 2007;133:1581-7.

20 Zheng W, Brown MD, Brock TA, et al. Bradycardia-induced coronary angiogenesis is dependent on vascular endothelial growth factor. Circ Res 1999:85:192-8.

21 Milasinovic D, Mohl W. Contemporary perspective on endogenous myocardial regeneration. World J Stem Cells 2015;7:793-805.

22 Syeda B, Schukro C, Heinze G, et al. The salvage potential of coronary sinus interventions: meta-analysis and pathophysiologic consequences. J Thorac Cardiovasc Surg 2004;127:1703-12.

23 Mohl W, Komamura K, Kasahara $\mathrm{H}$, et al. Myocardial protection via the coronary sinus. Circ J 2008;72:526-33.

24 Van de Hoef TP, Nolte F, Delewi R, et al. Intracoronary hemodynamic effects of pressure-controlled intermittent coronary sinus occlusion (PICSO): results from the First-In-Man Prepare PICSO Study. J Interv Cardiol 2012;25:549-56.

25 van de Hoef TP, Nijveldt R, van der Ent M, et al. Pressure-controlled intermittent coronary sinus occlusion (PICSO) in acute ST-segment elevation myocardial infarction: results of the Prepare RAMSES safety and feasibility study. Eurolntervention 2015;11:37-44.

26 Verheye S, Jolicœur EM, Behan MW, et al. Efficacy of a device to narrow the coronary sinus in refractory angina. N Engl J Med 2015;372:519-27.

27 Banai S, Ben Muvhar S, Parikh KH, et al. Coronary sinus reducer stent for the treatment of chronic refractory angina pectoris: a prospective, open-label, multicenter, safety feasibility first-in-man study. J Am Coll Cardiol 2007;49:1783-9.

28 De Maria GL, Patel N, Kassimis G, et al. Spontaneous and procedural plaque embolisation in native coronary arteries: pathophysiology, diagnosis, and prevention. Scientifica (Cairo) 2013;2013:364247.

29 Myat A, Patel N, Tehrani S, et al. Percutaneous circulatory assist devices for high-risk coronary intervention. JACC Cardiovasc Interv 2015;8:229-44.

30 Lazar HL, Yang XM, Rivers S, et al. Retroperfusion and balloon support to improve coronary revascularization. J Cardiovasc Surg (Torino) 1992;33:538-44. 